\title{
Functions, pathophysiology and current insights of exosomal endocrinology (Review)
}

\author{
DIMITRIOS VLACHAKIS ${ }^{1-3}$, THANASIS MITSIS ${ }^{1}$, NICOLAS NICOLAIDES ${ }^{2,4}$, ASPASIA EFTHIMIADOU ${ }^{5}$, \\ ANTONIS GIANNAKAKIS ${ }^{2,4}$, FLORA BACOPOULOU ${ }^{2}$ and GEORGE P. CHROUSOS ${ }^{2,3}$ \\ ${ }^{1}$ Laboratory of Genetics, Department of Biotechnology, School of Applied Biology and Biotechnology, \\ Agricultural University of Athens, 11855 Athens; ${ }^{2}$ University Research Institute of Maternal and Child Health and \\ Precision Medicine, and UNESCO Chair on Adolescent Health Care, National and Kapodistrian University of Athens, \\ 'Aghia Sophia' Children's Hospital, 11527 Athens; ${ }^{3}$ Division of Endocrinology and Metabolism, Center of Clinical, \\ Experimental Surgery and Translational Research, Biomedical Research Foundation of The Academy of Athens, \\ 11527 Athens; ${ }^{4}$ Department of Molecular Biology and Genetics, Health Sciences School, Democritus University of Thrace, \\ 68100 Alexandroupolis; ${ }^{5}$ Hellenic Agricultural Organization-Demeter, Institute of Soil and \\ Water Resources, Department of Soil Science of Athens, 14123 Lycovrisi, Greece
}

Received May 23, 2020; Accepted October 6, 2020

DOI: $10.3892 / \mathrm{mmr} .2020 .11664$

\begin{abstract}
Studies on extracellular vesicles have increased in recent years. The multi-dimensional nature of their roles in cellular homeostasis, cell-to-cell and tissue-to-tissue communication at the level of the organism, as well as their actions on the holobiome (intra-/interspecies interaction), have garnered the interest of a large number of researchers. Exosomes are one of the most researched classes of extracellular vesicles because they are carriers of targeted protein and DNA/RNA loads. Their multi-functional cargo have been indicated to regulate a vast number of biological pathways in target cells. However, the mechanisms governing these interactions have not yet been fully determined. Endocrinology, by definition, focuses on homeostatic, and cell-to-cell and tissue-to-tissue communication mechanisms. Therefore exosomes should be included in this research topic. Exosomes have previously been associated with a number of endocrine disorders, including
\end{abstract}

Correspondence to: Dr Dimitrios Vlachakis, Laboratory of Genetics, Department of Biotechnology, School of Applied Biology and Biotechnology, Agricultural University of Athens, Chasioti Building, 75 Iera Odos Street, 11855 Athens, Greece

E-mail: dimvl@aua.gr

Professor George P. Chrousos, University Research Institute of Maternal and Child Health and Precision Medicine, and UNESCO Chair on Adolescent Health Care, National and Kapodistrian University of Athens, 'Aghia Sophia' Children's Hospital, Choremeion Research Laboratory Building, Thivon and Livadias 8 Street, 11527 Athens, Greece

E-mail: chrousos@gmail.com

Key words: extracellular vesicles, exosomes, endocrine disorders, virus, medical applications obesity, type 2 diabetes mellitus, disorders of the reproductive system and cancer. Furthermore, their biogenesis, composition and function have been associated with viruses, an entirely different domain of life. The profound roles of exosomes in homeostasis, stress and several pathological conditions, in conjunction with their selective and cell-specific composition/function, allude to their use as promising circulating clinical biomarkers of systemic stress and specific pathologic states, and as biocompatible vehicles of therapeutic cargo. The current review provides information on exosomes and discusses their endocrine implications.

\section{Contents}

1. Introduction

2. An in-depth view of exosomes

3. Cell-to-cell interactions and the microenvironment

4. Biological processes

5. Disorders of the endocrine system and exosomes

6. Exosome-based personalized medical applications

7. Conclusion

\section{Introduction}

Exosomes are small lipid bilayer-surrounded extracellular vesicles released from cells into the extracellular space or biological fluids $(1,2)$. The biological fluids exosomes are found in abundance in include blood, saliva, milk, urine, semen, and bile juice (3). These vesicles are carriers of active or non-autonomous function biomolecules, such as proteins, lipids, DNA, mRNA and non-coding regulatory RNA. Exosomal markers include microRNAs like miR-21 and miR-141, plus various proteins that belong in functional groups such as tetraspanins (CD9, CD63 and CD81), heat shock 
proteins (Hsp70, Hsp73 and Hsp90) and membrane transporters (GTPases) (4). Exosomes, via their cargo or surface composition, are signals/mediators of systemic homeostasis and stress for specific cell-to-cell or tissue-to-tissue communication (5). They are an integral part of the later phase of the cellular stress response, i.e. the stress-induced senescence-like phenotype, as well as the senescence-associated secretory phenotype. Because of their expanding roles in the endocrine regulation of homeostasis and stress, and their involvement in human pathophysiology, exosomes are now the epicenter of a new field, termed 'exosomics' (3).

Extracellular vesicles (EVs) comprise a large spectrum of lipid bilayer-covered micro-particles; based on their size-range, they can be broadly classified into three distinct classes: microvesicles (MVs), exosomes and apoptotic bodies (ABs) (Fig. 1) (6). Microvesicles have a diameter ranging from $100 \mathrm{~nm}$ to $1 \mu \mathrm{m}$ and are released by cell membrane budding. Exosomes have a diameter in the range of 30-120 $\mathrm{nm}$. They are derived by a targeted mechanism from the cell endocytic compartment and are formed and stored within the intracellular multivesicular bodies (MVBs). Lastly, apoptotic bodies have a diameter from $50 \mathrm{~nm}$ to $5 \mu \mathrm{m}$, the latter a dimension similar to that of platelets $(2-3 \mu \mathrm{m})$. They originate from the blebbing of dying cells $(1,7,8)$.

Exosomes have been heavily researched in the past few years. Numerous technologies have been developed both for the isolation and characterization of these vesicles (4). Exosome isolation methods include differential centrifugation, density gradient centrifugation, size exclusion chromatography, ultrafiltration, polymer-based precipitation, immunological separation and isolation by sieving (4). The characterization technologies used for exosomes make use of biophysical methods, such as optical particle tracking that measures from $10 \mathrm{~nm}$ to $2 \mu \mathrm{m}$, molecular methods, such as Raman spectrometry that provides the chemical structure of the exosomes, and microfluidic methods that make use of immunoisolation and targeted protein analysis of circulating exosomes $(4,9)$. Exosomes appear to have major roles in both the physiology and pathophysiology of multicellular organisms (10). When they are released under physiological conditions, exosomes are essential for proper immune system function, while under pathological circumstances, they potentiate cellular stress and damage (11). Some of the diseases associated with exosomes are the cardiometabolic and neurodegenerative disorders and cancer (11). Finally, exosomes have a significant role in viral infections (12). Virus-infected cells release exosomes that contain a variety of viral and host cellular factors that can modify recipient host cell responses (12).

The research on exosomes is expanding the traditional view on endocrine and neuroendocrine signaling mechanisms. Traditionally, endocrine mechanisms include specialized groups of cells, the glands, their molecular mediators, which are secreted in the general circulation, i.e., the hormones and the distant target tissues of the latter (13). Hormones are unique chemical messengers that can be recognized by specific receptors in their target tissues (13). As exosomes carry bioactive molecules from donor cells and express surface proteins that allow them to transfer their contents into their target cells, they themselves or their cargo may function as 'hormones'. They can, thus, participate as such in an autocrine, i.e., local signals between the same type of cells, paracrine, i.e., local signals between different types of cells, or endocrine, i.e., distant signals between any type of cell reaching their target cells via the systemic or local circulation $(14,15)$.

Therefore, taking into consideration the involvement of exosomes in current endocrine research, we suggest that they can provide useful information and lead to a large number of potential clinical applications. Some of these applications include their use as biomarkers and as a novel method of message or drug delivery (16).

\section{An in-depth view of exosomes}

Biogenesis. The classification of EVs is mainly based on the mechanisms of their biogenesis. Exosome biogenesis begins within the endosomal system, apoptotic bodies are released by cells undergoing programmed death, i.e. apoptosis, while microvesicles are derived directly from plasma membrane blebbing (17). Early endosomes grow into late endosomes by acidification, changes in their protein content, and increased ability to fuse with other membranes. Late endosomes then form multivesicular bodies (MVBs) through reverse budding, during which the endosomal membrane invaginates to form intraluminal vesicles (ILVs) (18). Multivesicular bodies transport molecules that have been marked by mono-ubiquitination or by tetraspanins. The endosomal sorting complexes required for transport (ESCRT) mediate one of the main mechanisms of incorporation of molecules into ILVs (19). MVBs may then follow different pathways: i) directed to lysosomes for content degradation; ii) transported to the plasma membrane to fuse with it and release the ILVs outside the cell as exosomes; iii) directed to the plasma membrane to present antigen-loaded major histocompatibility complex (MHC) class II molecules; and iv) recycled (18-20).

It appears that different types of cells may be under conditions and/or exposed to factors that promote the release of some MVBs as exosomes. Apart from sorting through the aforementioned ESCRT, another possible mechanism is one in which cargo is sorted to specific lipid-raft-enriched microdomains that are associated with ILVs destined for exosome release (20). This possibility is supported by the fact that post-translational modifications, such as $\mathrm{N}$-glycosylation, direct proteins to specific microdomains on the limiting membrane of late endosomes to sort into ILVs also destined for exosome release (20).

Multivesicular bodies' transport within the cell and towards the cell membrane is based on interactions with actin and microtubes of the cytoskeleton (21). Many proteins partake in MVB fusion with the plasma membrane and subsequent exosome release. Specifically, the GTPase family of Rab proteins appears to be of high importance in regulating vesicle tethering and fusion (22). This protein family action on exosome release seems to be cell-specific. Different cells possibly use different Rab proteins to regulate exosome release in response to membrane fusion (21). Another group of proteins that may have an important role in membrane fusion and exosome secretion are the SNAP receptor proteins (SNARE), which are regulators of vesicle docking and fusion (22). The involvement of these proteins in the release of 


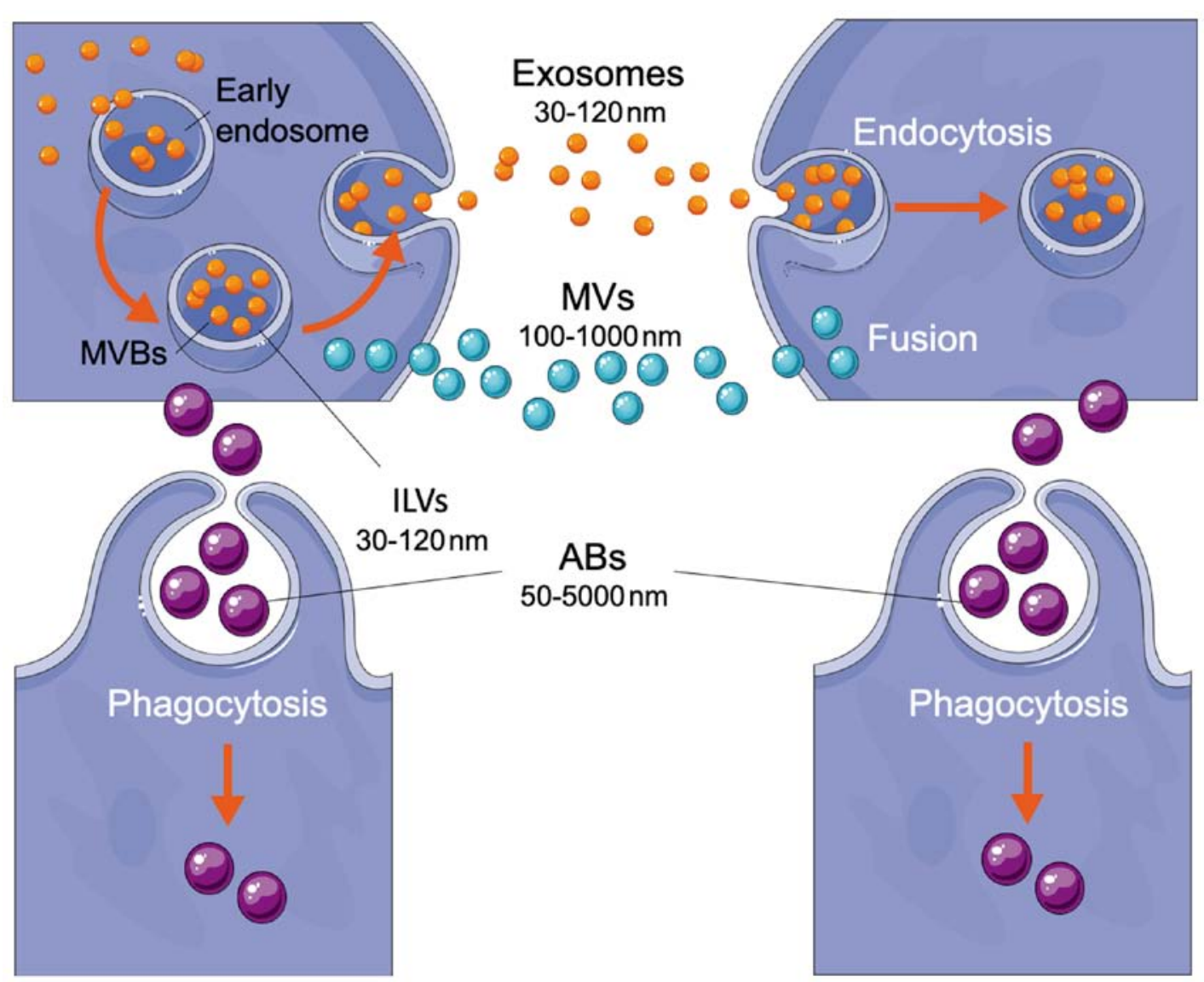

Figure 1. Biogenesis and classification of extracellular vesicles. AB, apoptotic bodies; MVBs, multivesicular bodies; MVs, microvesicles; ILVs, intraluminal vesicles.

the final exosomes in response to membrane fusion is through the formation of a SNARE complex composed of proteins containing three-fourth coiled-coil helices (21).

\section{Cell-to-cell interactions and the microenvironment}

As already mentioned, the role of exosomes as generic or specific carriers of signals and regulatory molecules through a pathway of intercellular vesicle traffic is one of the main reasons for the explosion of exosome research (23). After their release, a number of set events characterize exosome function. First, they undergo a change in their environment, as they move from the cytoplasm and cell surface to the extracellular fluid. Subsequently, exosomes interact with the plasma and endocytic membranes of target cells. Last, the ensuing fusions between exosomes and cell membranes complete the exosome signaling pathway (24).

All EVs showcase a number of common attributes. On release, some extracellular vesicles cannot remain intact, their membranes break down, and their bioactive cargo is expelled into the extracellular space. The released contents-which may include bioactive molecules, such as interleukin-1 $\beta$, growth factors, and tissue factors-bind their corresponding receptors in adjacent cells and activate rapid responses (25). Most EVs, though, maintain their structure and, therefore, survive in the extracellular fluid, even for long periods of time (24). Extracellular vesicles that persist in biological fluids may promote degradation of the extracellular matrix by their transmembrane proteases, an important mechanism for the circulation of macrophages and for tissue invasion by cancer cells (25). After their release, EVs can accumulate in the extracellular spaces close to intercellular junctions, moving between and through cells so they may leave the initial fluid and move to adjacent areas or tissues (24). Later on, these extracellular vesicles do not interact generally with any type of cell, but rather showcase a preference for specifically targeted cells (25). The mechanisms that dictate this ability of EVs are still under research.

A few mechanisms have been proposed specifically for exosome-mediated cell-to-cell interaction: i) exosome membrane proteins may interact with their corresponding membrane receptors on target cells, activating intracellular signaling; ii) exosome membrane proteins may be cleaved by proteases, and the resultant soluble fragments may act as ligands that bind to cell surface receptors; or iii) exosomes may be internalized by their target cells, releasing their cargo and activating downstream events within the receiving cells (26).

In the last-mentioned mechanism, exosome cargo delivery is responsible for the transport of genetic information between cells (27). Specifically, the RNA cargo of exosomes, including both mRNAs and noncoding (nc) RNAs, seems to be of high importance. This RNA appears to be functional in target cells and can modulate gene activity and/or protein production (28). Particularly, microRNAs, a class of regulatory small endogenous non-coding RNAs, have been thoroughly researched for their possible clinical applications (29-31). 


\section{Biological processes}

Exosome function and heterogeneity depend on the cell of origin and the conditions at the time of exosome generation in the originating tissues or cells (32). Exosomes partake in a wide number of biological processes, beyond inter-cellular communication. Some of these processes include angiogenesis, antigen presentation, inflammation, cellular homeostasis, apoptosis, and cell differentiation (32).

Angiogenesis. Angiogenesis is a process in which new blood vessels arise from preexisting vasculature (33). Microcirculation is vital for tissue homeostasis by regulating the supply of oxygen and nutrients and removing products of cellular metabolism. As exosomes can carry several biomolecules to target cells and alter their phenotype, it is not surprising that they can be carriers of pro- or anti-angiogenic signaling molecules (34). Exosomes derived by mesenchymal stem cells (MSC-derived exosomes) may carry microRNAs with angiogenic effect, such as miR-21 which activates the ACT and ERK pathways of the recipient cell and promotes vascular endothelial growth factor (VEGF), a signaling molecule which promotes the growth of new blood vessels (35). On the other hand, platelet-derived exosomes from human septic patients contain the $\mathrm{p} 22$ phox and gp91phox subunits of the NADPH oxidase, which is associated with endothelial cell apoptosis and dysfunction, thus showcasing anti-angiogenic effects (34). A pathological condition that exploits exosome role in angiogenesis is cancer. Cancer cells are in need of a constant supply of nutrients, oxygen, and growth factors. Angiogenesis provides new blood vessels that can supply cancer cells with the requirements mentioned above for survival. These blood vessels can promote tumor growth and metastasis formation. Exosomes seem to be mediators of such mechanisms because tumor cell-derived exosomes seem to promote angiogenesis (34).

Immunity. Exosomes secreted by antigen-presenting cells (APCs), such as dendritic cells (DCs), carry molecules involved in antigen presentation, for instance, MHC class I and II molecules and molecules involved in T-cell stimulation, such as CD86 (36). Particularly, antigens internalized by DCs and other professional APCs are processed into peptides and are incorporated into MHC class I (peptide-bound MHC I/pMHCI) or MHC class II (peptide-bound MHCII/pMHCII) molecules, which can later be transported to the extracellular space in extracellular vesicles such as exosomes (37). MHC class II molecules that are found on antigen-presenting cells-derived exosomes can stimulate CD4+ T-cells (36). Specifically, in the case of DC-derived exosomes, antigen-specific pMHCII found on the surface of the mentioned exosomes can activate the T-cell antigen receptor on the $\mathrm{CD} 4{ }^{+} \mathrm{T}$-cells and either stimulate them to proliferate directly or indirectly through the help of additional DCs. The need for additional DCs is dependent on the abundance of co-stimulatory molecules expressed in the exosome membrane (37). $\mathrm{CD}^{+} \mathrm{T}$-cells have an important role in adaptive immunity, where they mediate immune responses through the secretion of specific cytokines (38). MHC class I molecules found on APC-derived exosomes could potentially activate $\mathrm{CD}^{+} \mathrm{T}$-cells (36). Indeed, it has been shown that DC-derived exosome feature functional pMHCI molecules and can activate $\mathrm{CD}^{+} \mathrm{T}$-cell clones either alone or when incubated with DCs that express allogeneic MHC class I molecules (39). Naïve CD8 ${ }^{+}$T-cells can react to pathogens through expansion and differentiation into cytotoxic effector cells that can wander to all corners of the body to clear infections (40).

Inflammation. Exosome cargo has been shown to alter recipient cell functions, including changes in inflammatory responses (41). The above, along with the fact that different immune cells, such as macrophages, can both produce exosomes and receive mentioned vesicles, implies an important role for exosomes in inflammation (41). Exosomes have been associated with a number of pro-inflammatory cytokines, such as tumor necrosis factor-alpha (TNF- $\alpha$ ), interleukin-1 beta (IL-1 $\beta$ ) and interleukin-6 (IL-6), while they have also been associated with microRNAs showcasing anti-inflammatory actin such as miR-155 and miR-146a (41). Moreover, exosomes have also been associated with inflammation-related molecules, like small heat shock proteins (HSPs) (42). Some of these proteins are induced in response to stressful events, while others are expressed at a constant rate (42).

Apoptosis. The secretion of exosomes plays an essential role in maintaining cellular homeostasis of exosome-secreting cells (43). It is speculated that EVs may remove harmful cytoplasmic DNA from cells. The accumulation of such DNA causes activation of the cytoplasmic DNA-sensing machinery that promotes the innate immune response. This accumulation could lead to activation of reactive oxygen species (ROS)-dependent DNA damage response, resulting in cell cycle arrest and/or apoptosis in physiological human cells (43).

Apoptosis is an evolutionarily conserved mechanism of programmed cell death in multicellular organisms (44). These organisms need to tightly regulate their number of cells, which is achieved, among other mechanisms, through coupling of cell division and apoptosis control (45). Under normal circumstances, apoptosis helps maintain homeostasis by eliminating unwanted cells as a response to particular molecular signals. Apoptotic mechanisms are mainly characterized by condensation of chromatin material, DNA fragmentation in the nucleus, and cell shrinkage (46). Exosomes can induce cell cycle arrest and apoptosis in specific cells (32). A pathological condition making use of this exosomal ability includes exosomes derived from liver metastases of colorectal cancer. These exosomes can inhibit cell proliferation and induce T-cell apoptosis by carrying the Fas ligand (32). Moreover, apoptotic cells themselves release exosomes with specific attributes (47), through which they communicate with neighboring cells. These apoptotic cell-derived-exosomes seem to regulate inflammation and immune response $(47,48)$. Specifically, apoptotic cell-derived exosomes produced from endothelial cells carry ncRNAs, which can stimulate retinoic acid-inducible gene I-like receptors (RIG-I-like receptors) and toll-like receptors (TLRs) to promote inflammation, while apoptotic cell-derived exosomes produced from thymocytes induces TGF $\beta$ in macrophages and suppresses immune response (48).

Differentiation.Cell differentiation is a vital process in multicellular organisms and is involved in several biological functions, such as development, growth and reproduction (49). Exosomes, 
as significant mediators of intercellular communication, have been implied to participate in stem cell differentiation (50). A prime example is exosomes derived from cells cultured under osteogenic conditions (osteogenic exosomes), which can be endocytosed by human marrow-derived stromal cells (HMSCs) and lead to cell differentiation. Specifically, osteogenic exosomes lead to an increase in the expression levels of growth factors bone morphogenetic protein 9 (BMP9) and transforming growth factor $\beta 1$ (TGF $\beta 1$ ), which have been described as good inducers of osteogenic differentiation of MSCs (50).

Stress. Living organisms have to cope with various challenges during their lifespan. In order to cope with such challenges, living organisms are programmed to maintain an inner biological equilibrium, between an abiotic (chemical/physical) environment and the holobiome of all interacting life species. This inner equilibrium or homeostasis is vital for proper organism function (51). Unforeseen internal or external stimuli, the stressors, can threaten homeostasis, and the state of threatened, or perceived as such, homeostasis is called stress. Exposure to stressors induces a highly adaptive and integrated response system, the stress system, whose main goal is the re-establishment of homeostasis $(51,52)$. Precise communication among organs and cells is essential in maintaining homeostasis and responding to stressors. A system that includes precise communication mechanisms affiliated with the maintenance of homeostasis is the endocrine system (53). Exosomes are important mediators of inter-cellular communication and, therefore, partake in mechanisms related to homeostasis. These vesicles may act as important components of a complex endocrine system that provides endocrine, paracrine and autocrine signals (54).

Exosomes have a role in stress physiology (55). Supporting this concept is the fact that exosomes ubiquitously express membrane proteins that are associated with stress, like Hsp72, which also exert pro-inflammatory effects (56). It has been demonstrated that exposure to acute stressors in the absence of injury, inflammation, or disease can alter exosome composition to boost innate immunity (55). Such alterations may include changes in both exosome membrane proteins and the vesicular microRNA cargo.

These circulating stress-modified exosomes may have an immunomodulatory role (55). Evidence shows that in the absence of stress or pathology, exosomes generally downregulate immune processes. This may be due to their microRNA cargo, which includes molecules, such as mir-126, that exert anti-inflammatory effects (56). Stress exposure, on the other hand, increases the Hsp72 content and reduces the microRNA cargo of blood-borne exosomes, changing their role from immunosuppressive to immunostimulatory (56).

Cell-to-virus interaction. As mentioned above, exosomes have an essential role in viral pathogenesis and immunity (12). Under physiological conditions, exosomes can stimulate immune cells and present viral antigens in response to viral infection (12). In pathological conditions, viral infection alters the composition of exosomes and their functions (57). Viruses appear to co-opt exosome communication through a number of mechanisms that promote viral propagation. In the case of the hepatitis $\mathrm{C}$ virus ( $\mathrm{HCV}$ ), the viral genome can enter ILVs and be secreted later within exosomes, which, thus, act as infectious particles (58). In the case of other viruses, such as the Epstein-Barr virus (EBV), exosomes may carry viral proteins that promote infectivity (59). Furthermore, in EBV infections, exosomes derived from infected cells may carry host proteins that modulate the immune response and boost infectivity (60). Another mechanism used by the human immunodeficiency virus (HIV) involves the incorporation of viral nucleic acid in exosomes, which can improve and sustain the production of the HIV in infected cells (59). Last, exosomes derived from viruses, such as the herpes simplex virus 1 (HSV1), may carry microRNAs that eliminate production of host proteins associated with anti-viral responses (59).

An intriguing observation on the connection between exosomes and viruses is that they display a number of similarities in their formation, architecture and molecular mechanisms of action, including the biogenesis and transportation of functional nucleic acids and proteins (12). In regards to biogenesis, enveloped viruses, like HIV, make use of the ESCRT machinery to facilitate viral budding (61). Furthermore, as exosomes and viruses can deliver active biomolecules from one cell to another, they both have been implicated in playing a major role in evolution (62).

On the topic of evolution, exosomes may contain proteins encoded by endogenous retroviruses (ERVs), which are genetic remnants of ancient retroviral infections (23). None of the human endogenous retroviruses are truly functional, but some of the proteins they encode have evolved to execute physiological functions, while aberrant expression of such proteins has been associated with various diseases (23). Moreover, the presence of functional genetic material in exosomes and viruses, indicates that they may function as potential mediators of horizontal gene transfer, not only between cells of the same organism but also across different species (63). All of the above similarities provide an incentive to further research the connection between exosomes and viruses, both in an evolutionary and molecular manner.

\section{Disorders of the endocrine system and exosomes}

Dysregulation of the endocrine system has been associated with a large number of pathological conditions. Some of these include obesity, type 2 diabetes mellitus (T2DM), disorders of the reproductive system, and various forms of cancer, such as breast, testicular, and ovarian cancer (64). Exosomes have been implicated in many of these and other pathological conditions.

Obesity is a chronic disorder characterized by excessive accumulation of body fat that increases health risks (65). Exosomes have a role in obesity and its metabolic complications through their action as mediators of communication between adipose tissue, skeletal muscle, liver and immune cells (66). Studies have shown that adipose-derived exosome microRNAs are differentially expressed between lean and obese individuals (66). Furthermore, research in animal models of obesity indicates that treatment of lean mice with exosomes containing obesity-associated microRNA mimics, induces central obesity and hepatic steatosis (67). A suggested mechanism via which obesity leads to health complications is through the induction of inflammation in major organs, 
including adipose tissue and tissues of the cardiovascular system (66). Exosomes may play a role in these mechanisms, as they have both pro-inflammatory and anti-inflammatory properties $(55,56)$.

Type 2 diabetes mellitus is a metabolic disease that has been associated with both obesity and presence of exosomes (68). This disease is characterized by hyperglycemia, insulin resistance, and insulin deficiency (69). T2DM is associated with the metabolic syndrome, a condition related to obesity and characterized by insulin resistance, dyslipidemia, hypertension and blood hypercoagulability (70). Exosomes released from obesity-related adipose tissue contain different microRNA cargo compared to those secreted by healthy adipose tissue and seem to significantly decrease insulin sensitivity and glucose uptake (71). The mechanisms via which these abnormal exosomes influence T2DM may include activation of inflammation, downregulation of glucose transporter type 4, and disruption of insulin receptor signaling (72).

Exosomes have also been implicated in a number of reproduction-related pathologies, like endometriosis and the polycystic ovary syndrome (PCOS) (73). Endometriosis is a gynecological disorder characterized by the presence of tissue that resembles the endometrium outside the uterine cavity (74). In this condition, studies implicate stromal endometrial-derived exosomes, which modulate the immune surveillance response, and allow ectopic endometrial tissue to attach to peritoneal surfaces. This ectopic endometrial tissue may continue to release exosomes that help it evade the immune system and survive (75).

Polycystic ovary syndrome is a common gynecological metabolic and reproductive disorder associated with menstrual dysfunction, androgen excess and decreased fertility (76). Exosomes derived from human follicular fluid display differences in their microRNA cargo between PCOS patients and controls (77). Bioinformatic analyses indicate that these differences may result in alterations in amino acid biosynthesis and metabolism (77).

An increase in exosome release and alterations in their composition may promote cancer progression and metastases (78). Specifically, exosomes released from tumor cells seem to have an essential role in cancer. These exosomes induce oncogenesis, reprogram stromal cells, modulate the immune system, and, as already mentioned above, promote angiogenesis (79). Particularly, tumor-derived exosomes can carry oncogenic and anti-apoptotic molecules among cells, promoting oncogenesis (79). Additionally, tumor-derived exosomes play an essential role in transforming normal stromal cells to cancer-associated fibroblasts through transforming growth factor beta (TGF $\beta$ ) signaling (80). Cancer-associated fibroblasts are key regulators of the tumor microenvironment and can modulate cancer metastases, immune system function, production of growth factors, and angiogenesis (81). Last, tumor-derived exosomes can act directly on immune cells to produce immunosuppression (80).

\section{Exosome-based personalized medical applications}

Precision medicine's goal is to provide the appropriate treatment to the fitting patient at the right time. In precision medicine, molecular factors may be used to provide precise diagnosis and, thus, lead to a more accurate treatment (82). Exosomes can be considered such factors with some of their potential applications, including their use as biomarkers or therapeutic biomolecule carriers (83). Biomarkers are cellular, biochemical, or molecular parameters that can be objectively measured to serve as accurate indicators of a biological state (84).

The key attribute exosomes showcase that can be exploited in therapeutic applications is that their origin dictates their cargo and role in cell signaling $(85,86)$. Levels of exosome population subgroups can reflect many aspects of cell-to-cell communication, such as the state of cellular homeostasis, the targeted pathway and tissue, and the dynamics of the extracellular environment. Furthermore, exosome cargo contains a mixture of active signals and non-autonomous genetic information protected by the lipid bilayer of the vesicle. Finally, exosomes are highly stable and, thus, can regulate the stress response at a systemic level by storing and transferring enriched amounts of regulatory information, as well as markers of stress, while retaining a cell- and state-type of specificity for low abundant proteins and RNAs (86), which is related to their cell of origin and the function(s) that they mediate.

Exosomes can be used as therapeutic agents due to their exceptional biocompatibility and bio-specificity, both as vehicles and as cargo, especially when combined with patient-derived embryonic stem cells (hESCs) (87). A prime example of ESC-derived exosomes showcasing therapeutic abilities analogous to their cell of origin is that of mesenchymal stem cell derived exosomes (88). MSC-derived exosomes can be used in tissue injury repair and can reduce the inherent health risks related to administering viable cells (88). On the other hand, exosomes can be modified to deliver drugs and vaccines. A prime example is loading exosomes with anticancer agents, an approach that has been shown to have therapeutic effects in animal models (89). Last, it should be mentioned that the diverse abilities of exosomes should be taken into consideration when they are used as therapeutic agents, as they may have adverse effects on a patient's physiological mechanisms, including those of the immune system, intermediary metabolism, and others (90).

\section{Conclusion}

Exosomes have a vital and largely unknown role in inter-cellular signaling. These vesicles seem to partake in a large number of biological functions, while they have also been associated with several diseases. It is, therefore, not surprising that there has been a high interest in exosome research over the recent years. Endocrinology encompasses most branches of biology and medicine and we can now add exosomes as endocrine cell-to-cell communication components. Thus, exosomal endocrinology represents yet another aspect of endocrinology, an essential field of research that may help improve understanding, prognosis, diagnosis, and treatment of many diseases.

\section{Acknowledgements}

Not applicable. 


\section{Funding}

Funding was received from Microsoft Azure for Genomics Research (grant no. CRM:0740983), Amazon Web Services Cloud for Genomics Research (grant no. 309211522729), AdjustEBOVGP-Dx (grant no. RIA2018EF-2081; Biochemical Adjustments of native EBOV Glycoprotein in Patient Sample to Unmask target Epitopes for Rapid Diagnostic Testing), a European \& Developing Countries Clinical Trials Partnership (EDCTP2) under the Horizon 2020 'Research and Innovation Actions' DESCA.

\section{Availability of data and materials}

Not applicable.

\section{Authors' contributions}

DV and GPC conceived of the current article. DV, TM, NN, AE, AG, FB and GPC have all contributed to the writing, drafting, revising, editing and reviewing of the manuscript. All authors read and approved the final manuscript.

\section{Ethics approval and consent to participate}

Not applicable.

\section{Patient consent for publication}

Not applicable.

\section{Competing interests}

The authors declare that they have no competing interests

\section{References}

1. Doyle LM and Wang MZ: Overview of extracellular vesicles, their origin, composition, purpose, and methods for exosome isolation and analysis. Cells 8: 727, 2019.

2. Lawson C, Vicencio JM, Yellon DM and Davidson SM Microvesicles and exosomes: New players in metabolic and cardiovascular disease. J Endocrinol 228: R57-R71, 2016.

3. Patel GK, Khan MA, Zubair H, Srivastava SK, Khushman Md, Singh S and Singh AP: Comparative analysis of exosome isolation methods using culture supernatant for optimum yield, purity and downstream applications. Sci Rep 9: 5335, 2019.

4. Yakimchuk K: Exosomes: Isolation and characterization methods and specific markers. Mater Methods 5: 1450, 2015. // dx.doi.org/10.13070/mm.en.5.1450.

5. Maacha S, Bhat AA, Jimenez L, Raza A, Haris M, Uddin S and Grivel JC: Extracellular vesicles-mediated intercellular communication: Roles in the tumor microenvironment and anti-cancer drug resistance. Mol Cancer 18: 55, 2019.

6. Yanez-Mo M, Siljander PR, Andreu Z, Zavec AB, Borras FE, Buzas EI, Buzas K, Casal E, Cappello F, Carvalho J, et al: Biological properties of extracellular vesicles and their physiological functions. J Extracell Vesicles 4: 27066, 2015.

7. Villa F, Quarto R and Tasso R: Extracellular vesicles as natural, safe and efficient drug delivery systems. Pharmaceutics 11: 557, 2019.

8. Zarka R, Horev MB, Volberg T, Neubauer S, Kessler H, Spatz JP and Geiger B: Differential modulation of platelet adhesion and spreading by adhesive ligand density. Nano Lett 19: 1418-1427, 2019.

9. He M, Crow J, Roth M, Zeng Y and Godwin AK: Integrated immunoisolation and protein analysis of circulating exosomes using microfluidic technology. Lab Chip 14: 3773-3780, 2014.
10. Camussi G, Deregibus MC, Bruno S, Cantaluppi V and Biancone L: Exosomes/microvesicles as a mechanism of cell-to-cell communication. Kidney Int 78: 838-848, 2010.

11. Isola AL and Chen S: Exosomes: The messengers of health and disease. Curr Neuropharmacol 15: 157-165, 2017.

12. Crenshaw BJ, Gu L, Sims B and Matthews QL: Exosome biogenesis and biological function in response to viral infections. Open Virol J 12: 134-148, 2018.

13. Nussey SS and Whitehead SA: Endocrinology: An Integrated Approach: CRC Press, 2001.

14. Thery C, Ostrowski M and Segura E: Membrane vesicles as conveyors of immune responses. Nat Rev Immunol 9: 581-593, 2009.

15. Zduriencikova M, Gronesova P, Cholujova D and Sedlak J: Potential biomarkers of exosomal cargo in endocrine signaling. Endocr Regul 49: 141-50, 2015.

16. Qin J and Xu Q: Functions and application of exosomes. Acta Pol Pharm 71: 537-543, 2014.

17. Willms E, Cabañas C, Mäger I, Wood MJA and Vader P: Extracellular vesicle heterogeneity: Subpopulations, isolation techniques, and diverse functions in cancer progression. Front Immunol 9: 738, 2018.

18. Hessvik NP and Llorente A: Current knowledge on exosome biogenesis and release. Cell Mol Life Sci 75: 193-208, 2018.

19. Turegano-Lopez M, Santuy A, DeFelipe J and Merchan-Perez A: Size, shape, and distribution of multivesicular bodies in the juvenile rat somatosensory cortex: A 3D electron microscopy study. Cereb Cortex 30: 1887, 2020.

20. Tang Y and Dawn B: Mesenchymal Stem Cell Derived Exosomes: The Potential for Translational Nanomedicine. 1st edition. Academic Press, 2015.

21. Catalano M and O'Driscoll L: Inhibiting extracellular vesicles formation and release: A review of EV inhibitors. J Extracell Vesicles 9: 1703244, 2020.

22. Beer KB and Wehman AM: Mechanisms and functions of extracellular vesicle release in vivo: What we can learn from flies and worms. Cell Adh Migr 11: 135-50, 2017.

23. Pegtel DM and Gould SJ: Exosomes. Annu Rev Biochem 88: 487-514, 2019.

24. Meldolesi J: Exosomes and ectosomes in intercellular communication. Curr Biol 28: R435-R44, 2018.

25. Cocucci E and Meldolesi J: Ectosomes and exosomes: Shedding the confusion between extracellular vesicles. Trends Cell Biol 25: 364-372, 2015.

26. Urbanelli L, Magini A, Buratta S, Brozzi A, Sagini K, Polchi A, Tancini B and Emiliani C: Signaling pathways in exosomes biogenesis, secretion and fate. Genes (Basel) 4: 152-170, 2013.

27. de la Torre Gomez C, Goreham RV, Bech Serra JJ, Nann T and Kussmann M: 'Exosomics': A review of biophysics, biology and biochemistry of exosomes with a focus on human breast milk. Front Genet 9: 92, 2018

28. Valadi H, Ekstrom K, Bossios A, Sjostrand M, Lee JJ and Lotvall JO: Exosome-mediated transfer of mRNAs and microRNAs is a novel mechanism of genetic exchange between cells. Nat Cell Biol 9: 654-659, 2007.

29. Huang Y, Shen XJ, Zou Q, Wang SP, Tang SM and Zhang GZ: Biological functions of microRNAs: A review. J Physiol Biochem 67: 129-139, 2011.

30. Wang M, Yu F, Ding H, Wang Y, Li P and Wang K: Emerging function and clinical values of exosomal MicroRNAs in cancer. Mol Ther Nucleic Acids 16: 791-804, 2019.

31. Beuzelin D and Kaeffer B: Exosomes and miRNA-loaded biomimetic nanovehicles, a focus on their potentials preventing type-2 diabetes linked to metabolic syndrome. Frontiers in immunology 9: 2711, 2018.

32. Gurunathan S, Kang MH, Jeyaraj M, Qasim M and Kim JH: Review of the isolation, characterization, biological function, and multifarious therapeutic approaches of exosomes. Cells 8: 307, 2019.

33. Tahergorabi $\mathrm{Z}$ and Khazaei $\mathrm{M}$ : $\mathrm{A}$ review on angiogenesis and its assays. Iran J Basic Med Sci 15: 1110-1126, 2012.

34. Ribeiro MF, Zhu H, Millard RW and Fan GC: Exosomes function in pro- and anti-angiogenesis. Curr Angiogenes 2: 54-59, 2013.

35. Zimta AA, Baru O, Badea M, Buduru SD and Berindan-Neagoe I: The role of angiogenesis and pro-angiogenic exosomes in regenerative dentistry. Int J Mol Sci 20: 406, 2019.

36. Pawankar R, Holgate S and Rosenwasser L: Allergy Frontiers: Future Perspectives. Vol 6. Springer, 2010.

37. Leone DA, Rees AJ and Kain R: Dendritic cells and routing cargo into exosomes. Immunol Cell Biol, 2018 (Ahead of print). 
38. Luckheeram RV, Zhou R, Verma AD and Xia B: CD4 ${ }^{+} \mathrm{T}$ cells: Differentiation and functions. Clin Dev Immunol 2012: 925135, 2012.

39. Théry C, Ostrowski M and Segura E: Membrane vesicles as conveyors of immune responses. Nat Rev Immunol 9: 581-593, 2009.

40. Zhang $\mathrm{N}$ and Bevan MJ: CD8 ${ }^{+} \mathrm{T}$ cells: Foot soldiers of the immune system. Immunity 35: 161-168, 2011.

41. Chan BD, Wong WY, Lee MM, Cho WC, Yee BK, Kwan YW and Tai WC: Exosomes in inflammation and inflammatory disease. Proteomics 19: e1800149, 2019.

42. Reddy VS, Madala SK, Trinath J and Reddy GB: Extracellular small heat shock proteins: Exosomal biogenesis and function. Cell Stress Chaperones 23: 441-454, 2018.

43. Takahashi A, Okada R, Nagao K, Kawamata Y, Hanyu A, Yoshimoto S, Takasugi M, Watanabe S, Kanemaki MT, Obuse C and Hara E: Exosomes maintain cellular homeostasis by excreting harmful DNA from cells. Nat Commun 8: 15287, 2017.

44. Singh R, Letai A and Sarosiek K: Regulation of apoptosis in health and disease: The balancing act of BCL-2 family proteins. Nat Rev Mol Cell Biol 20: 175-193, 2019.

45. Alberts B: Molecular biology of the cell, 6th edition. CRC Press, 2017.

46. Jan R and Chaudhry GE: Understanding apoptosis and apoptotic pathways targeted cancer therapeutics. Adv Pharm Bull 9: 205-218, 2019.

47. Caruso S and Poon IKH: Apoptotic cell-derived extracellular vesicles: More than just debris. Front Immunol 9: 1486, 2018.

48. Kakarla R, Hur J, Kim YJ, Kim J and Chwae YJ: Apoptotic cell-derived exosomes: Messages from dying cells. Exp Mol Med 52: 1-6, 2020.

49. Sanchez Alvarado A and Yamanaka S: Rethinking differentiation: Stem cells, regeneration, and plasticity. Cell 157: 110-119, 2014.

50. Narayanan R, Huang CC and Ravindran S: Hijacking the cellular mail: Exosome mediated differentiation of mesenchymal stem cells. Stem Cells Int 2016: 3808674, 2016.

51. Chrousos GP: Stress and disorders of the stress system. Nat Rev Endocrinol 5: 374-381, 2009.

52. Nicolaides NC, Kyratzi E, Lamprokostopoulou A, Chrousos GP and Charmandari E: Stress, the stress system and the role of glucocorticoids. Neuroimmunomodulation 22: 6-19, 2015.

53. Hiller-Sturmhofel S and Bartke A: The endocrine system: An overview. Alcohol Health Res World 22: 153-164, 1998.

54. Zhang HG and Grizzle WE: Exosomes: A novel pathway of local and distant intercellular communication that facilitates the growth and metastasis of neoplastic lesions. Am J Pathol 184: 28-41, 2014.

55. Beninson LA and Fleshner M: Exosomes: An emerging factor in stress-induced immunomodulation. Semin Immunol 26 394-401, 2014

56. Fleshner $M$ and Crane CR: Exosomes, DAMPs and miRNA: Features of stress physiology and immune homeostasis. Trends Immunol 38: 768-776, 2017.

57. Anderson M, Kashanchi F and Jacobson S: Role of exosomes in human retroviral mediated disorders. J Neuroimmune Pharmacol 13: 279-291, 2018.

58. Anderson MR, Kashanchi $\mathrm{F}$ and Jacobson S: Exosomes in viral disease. Neurotherapeutics 13: 535-546, 2016.

59. Urbanelli L, Buratta S, Tancini B, Sagini K, Delo F, Porcellati S and Emiliani C: The role of extracellular vesicles in viral infection and transmission. Vaccines (Basel) 7: 102, 2019.

60. Keryer-Bibens C, Pioche-Durieu C, Villemant C, Souquere S, Nishi N, Hirashima M, Middeldorp J and Busson P: Exosomes released by EBV-infected nasopharyngeal carcinoma cells convey the viral latent membrane protein 1 and the immunomodulatory protein galectin 9. BMC Cancer 6: 283, 2006.

61. Votteler J and Sundquist WI: Virus budding and the ESCRT pathway. Cell Host Microbe 14: 232-241, 2013.

62. Nolte-'t Hoen E, Cremer T, Gallo RC and Margolis LB: Extracellular vesicles and viruses: Are they close relatives? Proc Natl Acad Sci USA 113: 9155-9161, 2016.

63. Kawamura Y, Yamamoto Y, Sato TA and Ochiya T: Extracellular vesicles as trans-genomic agents: Emerging roles in disease and evolution. Cancer Sci 108: 824-830, 2017.

64. Maqbool F, Mostafalou S, Bahadar H and Abdollahi M: Review of endocrine disorders associated with environmental toxicants and possible involved mechanisms. Life Sci 145: 265-273, 2016.

65. Agha $M$ and Agha R: The rising prevalence of obesity: part A: Impact on public health. Int J Surg Oncol (N Y) 2: e17, 2017.
66. Kim A, Shah AS and Nakamura T: Extracellular Vesicles: A potential novel regulator of obesity and its associated complications. Children (Basel) 5: 152, 2018.

67. CastañoC,Kalko S, Novials A and Párrizas M:Obesity-associated exosomal miRNAs modulate glucose and lipid metabolism in mice. Proc Natl Acad Sci USA 115: 12158-12163, 2018.

68. Zhang B, Yang Y, Xiang L, Zhao Z and Ye R: Adipose-derived exosomes: A novel adipokine in obesity-associated diabetes. J Cell Physiol 234: 16692-16702, 2019.

69. Olokoba AB, Obateru OA and Olokoba LB: Type 2 diabetes mellitus: A review of current trends. Oman Med J 27: 269-273, 2012.

70. Nath D, Heemels MT and Anson L: Obesity and diabetes. Nature 444: 839, 2006.

71. Dang SY, Leng Y, Wang ZX, Xiao X, Zhang X, Wen T, Gong HZ, Hong A and Ma Y: Exosomal transfer of obesity adipose tissue for decreased miR-141-3p mediate insulin resistance of hepatocytes. Int J Biol Sci 15: 351-368, 2019.

72. Xiao Y, Zheng L, Zou X, Wang J, Zhong $\mathrm{J}$ and Zhong T: Extracellular vesicles in type 2 diabetes mellitus: Key roles in pathogenesis, complications, and therapy. J Extracell Vesicles 8: $1625677,2019$.

73. Simon C, Greening DW, Bolumar D, Balaguer N, Salamonsen LA and Vilella F: Extracellular vesicles in human reproduction in health and disease. Endocr Rev 39: 292-332, 2018.

74. Zondervan KT, Becker CM, Koga K, Missmer SA, Taylor RN and Vigano P: Endometriosis. Nat Rev Dis Primers 4: 9, 2018.

75. Schjenken JE, Panir K, Robertson SA and Hull ML: Exosome-mediated intracellular signalling impacts the development of endometriosis-new avenues for endometriosis research. Mol Hum Reprod 25: 2-4, 2018.

76. El Hayek S, Bitar L, Hamdar LH, Mirza FG and Daoud G: Poly cystic ovarian syndrome: An updated overview. Front Physiol 7: 124,2016

77. Hu J, Tang T, Zeng Z, Wu J, Tan X and Yan J: The expression of small RNAs in exosomes of follicular fluid altered in human polycystic ovarian syndrome. PeerJ 8: e8640, 2020.

78. Rajagopal $\mathrm{C}$ and Harikumar KB: The origin and functions of exosomes in cancer. Front Oncol 8: 66, 2018.

79. Tai YL, Chen KC, Hsieh JT and Shen TL: Exosomes in cancer development and clinical applications. Cancer Sci 109: 2364-3674, 2018

80. Tung KH, Ernstoff MS, Allen C an Shu SL: A Review of Exosomes and their Role in The Tumor Microenvironment and Host-Tumor 'Macroenvironment'. J Immunol Sci 3: 4-8, 2019.

81. Sahai E, Astsaturov I, Cukierman E, DeNardo DG, Egeblad M, Evans RM, Fearon D, Greten FR, Hingorani SR, Hunter T, et al: A framework for advancing our understanding of cancer-associated fibroblasts. Nat Rev Cancer 20: 174-186, 2020.

82. Currie G and Delles C: Precision medicine and personalized medicine in cardiovascular disease. Adv Exp Med Biol 1065: 589-605, 2018

83. Zhang Y, Liu Y, Liu H and Tang WH: Exosomes: Biogenesis, biologic function and clinical potential. Cell Biosci 9: 19, 2019.

84. Mayeux R: Biomarkers: Potential uses and limitations. NeuroRx 1: 182-188, 2004.

85. Sancho-Albero M, Navascués N, Mendoza G, Sebastián V, Arruebo M, Martín-Duque P and Santamaría J: Exosome origin determines cell targeting and the transfer of therapeutic nanoparticles towards target cells. J Nanobiotechnology 17: 16, 2019.

86. Boukouris S and Mathivanan S: Exosomes in bodily fluids are a highly stable resource of disease biomarkers. Proteomics Clin Appl 9: 358-367, 2015.

87. Liu C and Su C: Design strategies and application progress of therapeutic exosomes. Theranostics 9: 1015-1028, 2019.

88. Zhao T, Sun F, Liu J, Ding T, She J, Mao F, Xu W, Qian H and Yan Y: Emerging role of mesenchymal stem cell-derived exosomes in regenerative medicine. Curr Stem Cell Res Ther 14: 482-494, 2019.

89. Luan X, Sansanaphongpricha K, Myers I, Chen H, Yuan H and Sun D: Engineering exosomes as refined biological nanoplatforms for drug delivery. Acta Pharmacol Sin 38: 754-763, 2017.

90. Conlan RS, Pisano S, Oliveira MI, Ferrari M and Mendes Pinto I: Exosomes as reconfigurable therapeutic systems. Trends Mol Med 23: 636-650, 2017. 